\title{
Gamma Ray Effects on Trophozoites of Entamoeba histolytica
}

\author{
Curt R. Schneider ${ }^{1}$ and Richard J. Porter
}

\author{
School of Public Health, University of Michigan, Ann Arbor, Michigan
}

(Submitted for publication, 13 February 1959)

\begin{abstract}
Cultures of Entamoeba histolytica were subjected to pure gamma radiation from a cobalt-60 source. No effect upon population growth was noticed following dosages below 100,000 RAD. A noticeable increase in lag phase was observed following exposure to about 100,000 RAD. A dose of more than 195,300 RAD resulted in death as indicated by failure of cultures to grow. The relation between increasing lag phase and increasing dosages of radiation is represented by a logarithmic straight line.
\end{abstract}

Because of the paucity of standardized methods for experimenting with the entozoic amebae, information about their response to radiation has been scanty. Nasset and Kofoid (1928) incubated radium needles with cultures of Entamoeba histolytica (their Endamoeba dysenteriae) and recorded stimulation of in vitro growth. The radiation was composed, for practical purposes, of gamma rays but the level of intensity of ionization must have been comparatively low, for continuous exposures over 12-hour intervals failed to affect the rate of division adversely.

The single other reference to the radiosensitivity of parasitic amebae is the work of Sadun et al. (1950), who found that 120,000 roentgens of X-rays failed to inactivate completely amcbae growing in Shaffer-Fryc or Balamuth media. The normal cycle of population growth was not visibly affected by exposures up to 30,000 roentgens, but exposures of 60,000 and 120,000 roentgens seemed to inhibit growth.

In the following study an attempt has been made to amplify the work of Sadun et al. and to identify the level of ionizing radiation which is lethal for $E$. histolytica. The radiation consisted of pure gamma rays and dosages are reported in terms of $\mathrm{RAD}$ (corresponding to 100 ergs per gram).

1 Present address: Division of Medical Sciences, National Academy of Sciences - National Research Couneil, Washington, 1). C. Part of this work was accomplished under a contract with the U. S. Army Medical Department.

\section{Materials And Methods}

\section{Amebae}

The UC strain of $E$. histolytica was employed. This was obtained through the courtesy of Dr. Paul E. Thompson, Parke, Davis and Company, Detroit, Michigan, in 1950. It was isolated by Dr. Enid Rodaniche at the University of Chicago and is of uncertain origin. It produced no cysts during routine culture. The sediment of a single tube produced as many as $10^{6}$ organisms after 48 hours of growth. Guinea pigs could not be infected but erythrocytes were readily ingested in vitro. The associated bacterial flora is complex and is known to include Proteus vulgaris and several coliform organisms. Cultures were maintained in Ringer-egg-Locke slants. Rice starch $^{2}$ was routinely added in $10-20 \mathrm{mg}$ amounts to new cultures at the time of transfer. Horse serum was not employed.

\section{Radiation Source}

A source consisting of approximately 3 kilocuries of artificially radioactive cobalt $\left(\mathrm{Co}^{60}\right)$ provided pure gamma rays. A description of this source, in the Fission Products Laboratory, University of Michigan, and an analysis of its radiation field have been published by Lewis et al. (1954). Briefly, the source contains one hundred 10 -inch $\mathrm{Co}^{60}$ rods encased in aluminum and mounted in a

${ }^{2}$ Provided by the Stein-Hall Company, New York, N. Y. 
cylindrical holder in three concentric rows. 'The cylinder rides an elevator platform which can be lowered, for storage, to the bottom of a 17-foot well of water. For use, the carrier is raised into the radiation "cave," a room formed by concrete walls 4-feet thick. A special concave tube-holding rack was designed and used to ensure uniformity of distance of amebae from the source upon repeated exposures. Dosage was equated directly with distance from the source and with time.

\section{Irradiation Procedure}

For each radiation experiment, a pool of amebae was prepared from several 48-hour

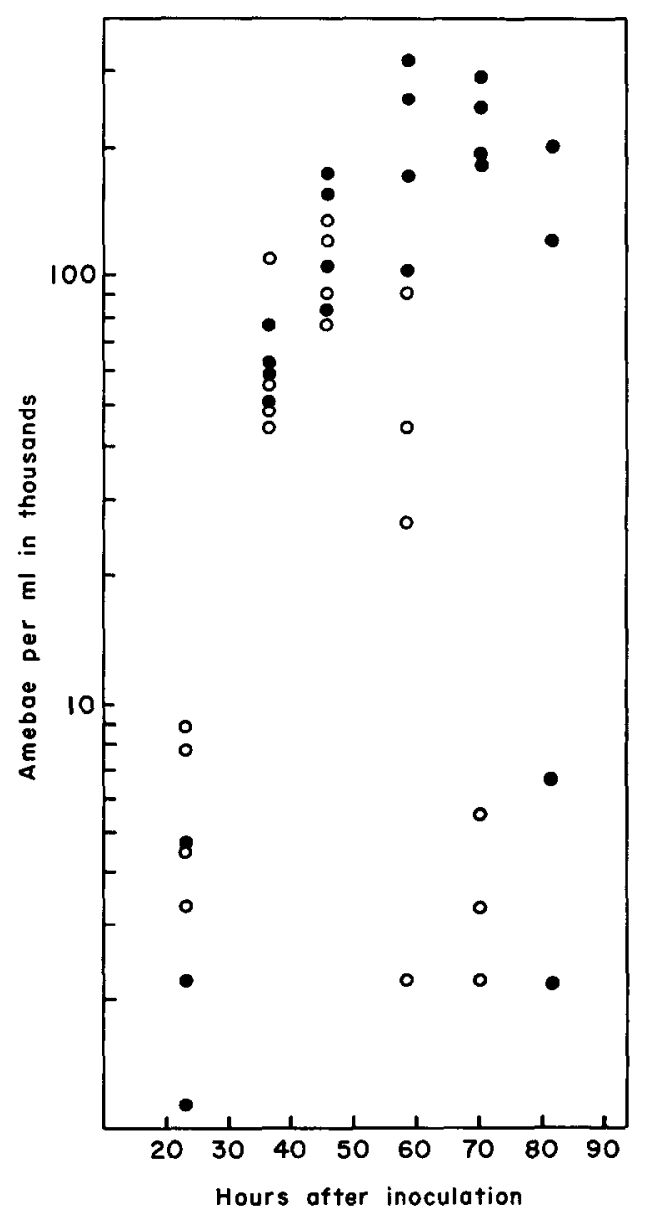

Fig. 1. Comparison of amebic growth following the addition of large and small amounts of rice starch to the cultures.

$O=10$ to $20 \mathrm{mg}$ added

$=35$ to $50 \mathrm{mg}$ added cultures. The pool was washed twice in sterile Stone's (1935) Locke solution to remove as many bacteria as possible. The resulting suspension of trophozoites was counted in a Spencer Bright-line hemocytometer according to Paulson's (1932) method, and adjusted to bring the count to between 20,000 and 30,000 organisms per milliliter. The counted suspension was then distributed in $1.5 \mathrm{ml}$ amounts to Lusteroid tubes (1/2 $\times 3 \frac{1}{2}$ inch) equipped with metal screw-caps. These tubes were placed in the special tube-rack at the requisite distance from the source and exposed to dosages ranging from 125,550 to 195,300 RAD. Control suspensions in all experiments were placed in the labyrinthine entrance to the radiation cave, where gamma radiation was negligible and where other conditions approximated those within the cave.

\section{Growth Determination}

At first, complete growth curves for each REL tube were found by counting at 8-hour intervals. Subsequently, tubes were observed until a single ameba could be counted in the hemocytometer and this time was arbitrarily defined as constituting the end of the lag phase of growth.

\section{EXPERIMENTAL}

\section{Effect of Rice Starch}

That an excess of rice starch can prolong the lag phase was recognized by Spingarn and Edelman (1950). Accordingly, preliminary tests were performed to ascertain the magnitude of increase in lag phase that would be produced by adding the largest amount of rice starch feasible under the conditions of the experiments. This amount was about 35 to $50 \mathrm{mg}$ per tube, or, the largest amount which could be heaped conveniently on the platinum spatula used routinely in rice-starch manipulations.

Standard inocula of washed amebae were introduced into eight preconditioned tubes. Four of these received 35 to $50 \mathrm{mg}$ of rice starch each. Controls received the amount of rice starch employed customarily, or, from 10 to $20 \mathrm{mg}$ per tube.

It will be seen from Fig. 1 that the populations of all tubes grouped themselves together during the period of logarithmic in- 


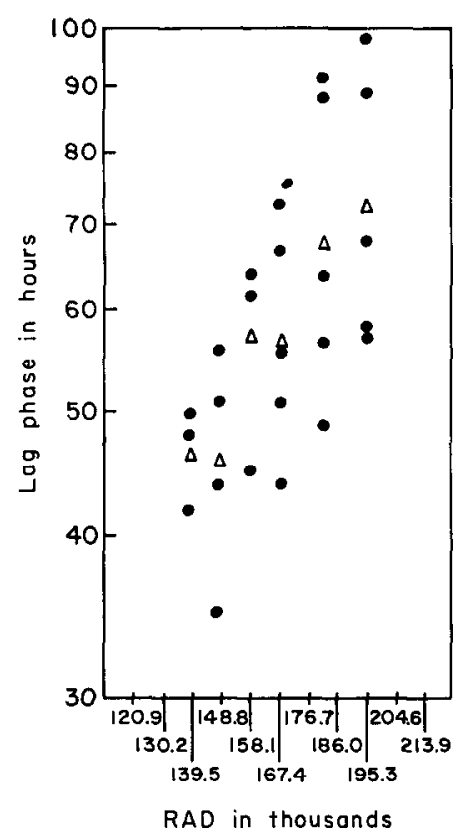

FIg. 2. Relation of length of lag phase of amebic growth to increasing doses of gamma radiation. $\triangle=$ antilog mean

crease. Thereafter, tubes with more starch maintained their peaks for about 10 hours longer than tubes with less starch. Spingarn and Edelman (1950) reported different absolute values for the same phenomenon.

Because of the uniformity in population growth, regardless of the amount of rice starch added, it was decided that "routine variation" in the amount of rice starch in the growth tubes probably would not affect the lag phase. Spingarn and Edelman (1950) prolonged the lag phase markedly with the addition of 200 to $400 \mathrm{mg}$ of rice starch, amounts notably in excess of those used here.

\section{Irradiation of Trophozoites}

Amebae were exposed, over a 6-month period, to varying dosages of radiation. Intensities in excess of 195,300 RAD always resulted in failure of the irradiated amehae. to develop in culture. The results of exposures ranging from 125,600 to 195,300 RAD are reported here. During each experiment, multiple exposures were made at the same dosage. Control suspensions were effectively shielded from gamma radiation by placing them in the labyrinthine entrance to the

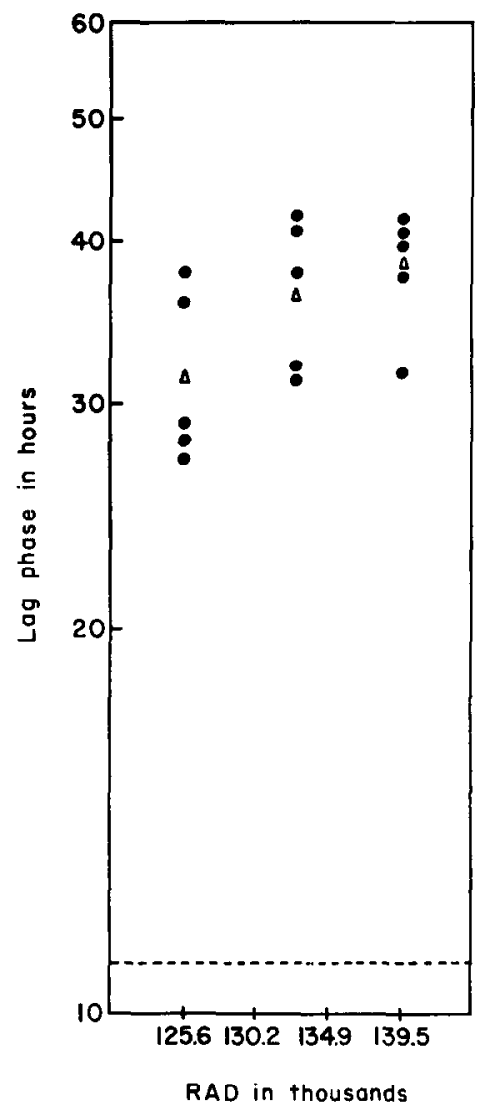

FIG. 3. Relation of length of lag phase of amebic growth to increasing doses of gamma radiation. Data obtained from simultaneous exposures at three different distances from the source.

cave. After irradiation each tube was cultured separately in the manner described previously and counts were made at 8-hour intervals to determine the end of the lag phase. Results are displayed in Figs. 2 and 3. Figure 2 represents values collected from many different experiments performed at different times during the 6-month period. Figure 3 shows values (not included in Fig. 2) from an experiment in which all tubes were irradiated at the same time but at different dosages. Plotting the points on semilogarithmic paper produced a scatter graph through which a striaght line may be fitled by eye.

These data enforce the conviction that the rate of inactivation of amebae is an exponential function of the dosage.

\section{Discussion}

Amebae were never found able to establish a population when irradiated with dos- 
ages of more than 195,300 RAD. Also, the lag phases of populations of amebae receiving $100,000 \mathrm{RAD}$ or less were indistinguishable from those of the controls. Thus, there was a relatively small range between the dosage at which no effect could be observed and that at which no amebae survived. Within this range the proportion of amebae unable to recover increascd logarithmically with time. It should be remarked that, although no growth was obtained following dosages greater than 195,300 RAD this may yet not represent the dosage at which all amebae were irreversibly damaged but one at which the survivors, if any, were few enough to be unable to reestablish themselves. Brackett and Bliznick (1947) found that as few as 500 amebae in association with organism $t$ produced positive cultures, and occasionally as few as fifteen were sufficient. It seems likely, then, that energy of 195,300 RAD approaches very nearly the true lethal dose of gamma rays for trophozoites of $E$. histoloytica.

\section{REFERENCES}

BracketT, S., ANd Bliznick, A. 1947. The rate of multiplication of Endamoeba histolytica and its relation to in vitro drug testing and possibly to nutritional studies. J. Parasitol. 33, 154-166.

Lewis, J. G., Nehemias, J. V., Harmer, D. E., and Martin, J. J. 1954. Analysis of radiation fields of two gamma-radiation sources. $\mathrm{Nu}$ cleonics 12, 40-44.

Nasset, E. C., And Kofold, C. A. 1928. The effects of radium and radium in combination with metallic sensitizers on Endamocba dysenteriae in vitro. Univ. Calif. Pubs. Zool. 31(17), 387416.

Paulson, M. 1932. An accurate method for the numerical determination of Entamoeba histolytica in vitro and its possible use with other intestinal prolozou. Am. J. Trop. Med. 12, $387-400$.

Sadun, E. H., Ané, J. N., Fuller, F. W., ANd LEWIS, R. 1950. The effect of X-ray upon the growth and infectivity of Endamoeba histolytica. Am. J. Trop. Med. 30, 635-640.

Spingarn, C. L., and Edelman, M. H. 1950. The effect of rice powder on the growth of cultures of Endamoeba histolytica. Am. J. Trop. Med. 30, 629-634. 